\title{
PARTICLE TRANSPORT, DEPOSITION AND REMOVAL- A COMBINED RESEARCH AND CURRICULUM DEVELOPMENT PROJECT
}

\author{
Goodarz Ahmadi, ${ }^{1}$ David J, Schmidt, ${ }^{1}$ Kambiz Nazridoust, ${ }^{1}$ Xiangwei Liu, ${ }^{1}$ Jeffrey \\ Taylor, ${ }^{1}$ Suresh Dhaniyala, ${ }^{1}$ John McLaughlin, ${ }^{1}$ Cetin Cetinkaya, ${ }^{1}$ Stephen Doheny- \\ Farina, ${ }^{1}$ and Fa-Gung Fan ${ }^{2}$ \\ ${ }^{1}$ Clarkson University, Potsdam, NY, 13699-5725 \\ ${ }^{2}$ Xerox Corporation, Webster, NY, 14580
}

\begin{abstract}
A sequence of two on particle transport, deposition and removal and reentrainment are described. The primary objective of these courses are to bring the new important research findings in the related field available to the class room for the benefit of seniors and first year graduate students in engineering. The course materials were also made available on the web and the courses were taught it at two campuses simultaneously.

\section{Introduction}

Understanding particle transport, deposition and removal are of crucial importance to many technologies that are critical for the competitiveness of the US microelectronic, imaging and pharmaceutical industries. In addition, solving a number of environmental problems requires a detail understanding of particle transport processes. In the last decade, significant research progress in the areas of particle transport, deposition and removal has been made. The primary objective of this combined research and curriculum development project is to make the fruits of these new important research findings available to seniors and first year graduate students in engineering through developing and offering of sequence of specialized courses. In these courses the process of particle transport, deposition and removal and re-entrainment was described. An extensive web for the course materials was developed and the courses were taught simultaneously at Clarkson University and Syracuse University.
\end{abstract}

\section{Course Modules}

These combined research and curriculum development (CRCD) courses are composed of four modules. The models are:

- Fundamental of particle transport, dispersion, deposition and removal.

- Computational modeling of particle transport, deposition and removal.

- Experimental study of particle transport, deposition and removal.

- Industrial applications of particle transport, deposition and removal. 
The front page of the course web is shown in Figure 1. The lecture notes and the calculations models are uploaded into the course web and are available in both pdf form as well as html form.

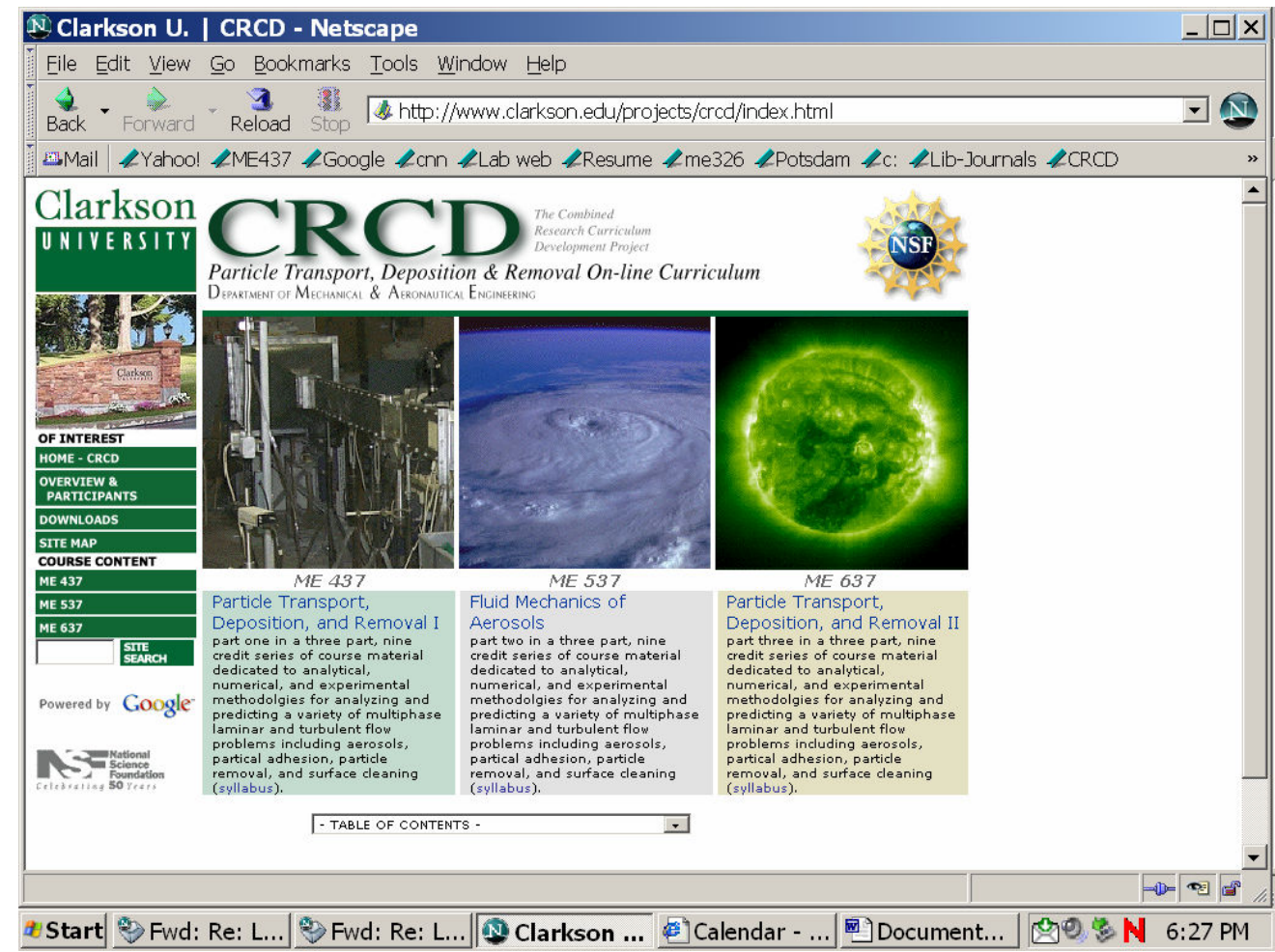

Figure 1. Front webpage of CRCD and the related courses.

\section{Module I, Fundamentals}

In Module I the descriptions of fundamentals of aerosols including hydrodynamic forces (drag, lift), and adhesion forces were described. The nature of particle adhesion and removal was also discussed. This module also contains the description of particle interaction with laminar flow, Brownian motion process, and particle deposition by diffusion, interception and impaction.

The sections on interaction of particles with turbulence and turbulent deposition that are normally taught in the second course. Computational modeling of turbulent flows was discussed, and classical models of turbulent deposition were described. In addition the process of aerosol charging and transport under the action of electrical forces and turbulence were discussed.

We have added a number of computational modules to make the course presentations of the materials more interactive. The plan is to have sufficient number of calculation modules for the student to experiment with. As a result the student will develop a physical understanding of some of the more complex concepts. 


\section{Module II, Computer Simulations}

We refined and developed several computer modules that were incorporated into the course sequence. One class of examples was concerned with exploring the flow and particle transport in a variety obstructed ducts. Fortran simulation programs that were developed in earlier were converted to JAVA. These programs were incorporated in the modules dealing with the motion of aerosol particles in the obstructed duct flows. The students will be able to interactively use the programs to explore the effects of various forces (gravity, drag, lift, Brownian), materials properties (particle density), and the flow geometry on the motion and deposition of particles.

A module was developed for illustrating Brownian particle motion in cross flows. The flow field in this module is a parabolic velocity profile between two parallel plates. The particle equation of motion includes Brownian motion, drag, lift, and gravity. Figure 2 shows the user interface for this module. Here, particles are injected from a nozzle in the middle of the channel. Here the dispersion of the Brownian particles can be seen. The module can also be used to illustrate the effects of the lift force on larger particles. Student can select values of the particle diameter and density, the number of particles, and the centerline fluid velocity.

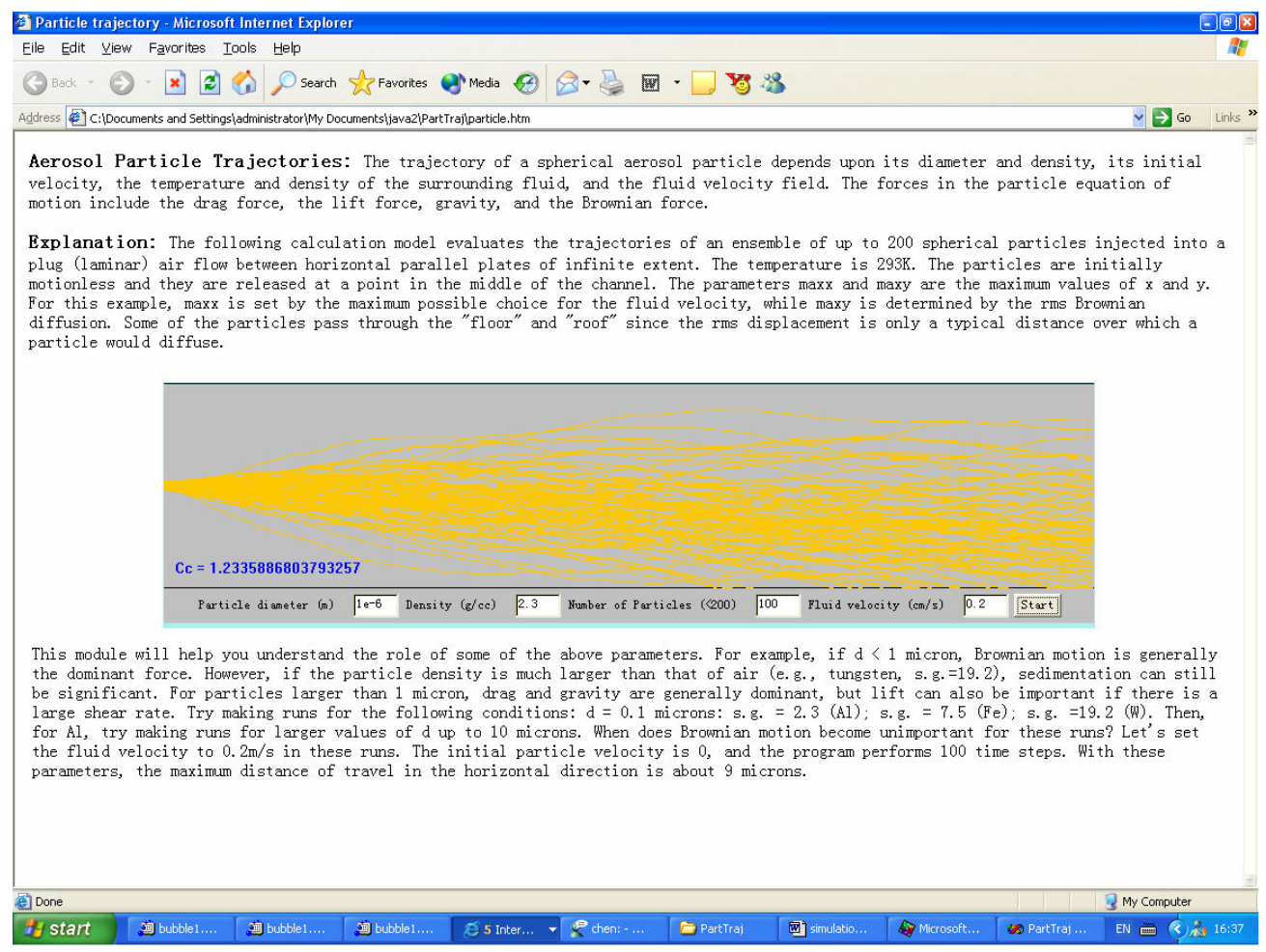

Figure 2. User interface for the module for Brownian particle motions in cross flows.

\section{Module III, Experimental}

The course sequence includes several experimental modules. One main experiment is the measurement in the aerosol wind tunnel with the use of Particle Image Velocimeter (PIV). The aerosol wind tunnel is located in the Turbulence and Multiphase 
Flow Laboratory at Clarkson University. The laser used was a $120 \mathrm{~mJ} \mathrm{Nd}$ :YaG laser with a $20^{\circ}$ adjustable width sheet generator. In this experiment, the sheet width was 0.5 $\mathrm{mm}$. The digital camera that was used was a Kodak ES1.0 MegaPlus camera. The camera had a pixel range of $1008 \times 1008$. The pixel size was 25 micrometers and the interframe delay between pictures was 12 microseconds. A picture of the experimental setup is show in Figure 3. A sample PIV measurement of the velocity field behind a step is shown in Figure 4.

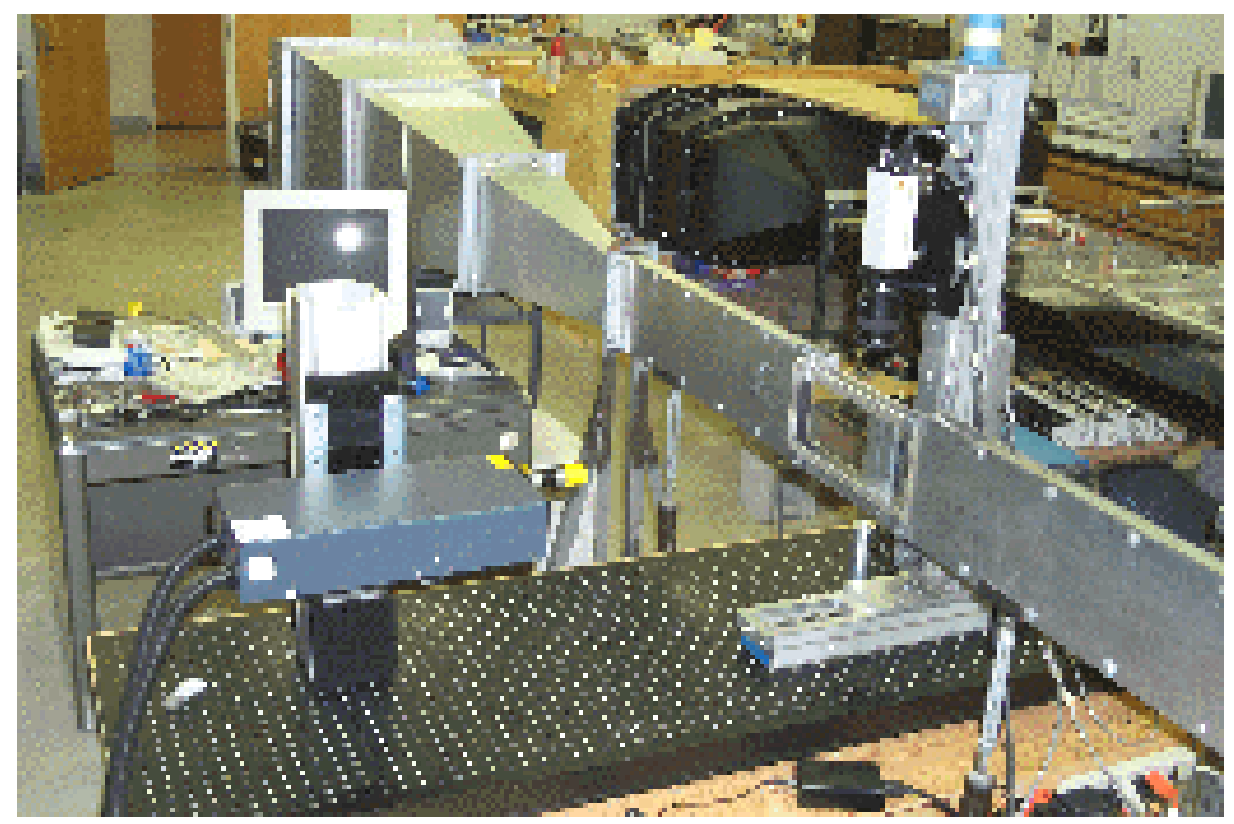

Figure 3. A picture of the aerosol wind tunnel.

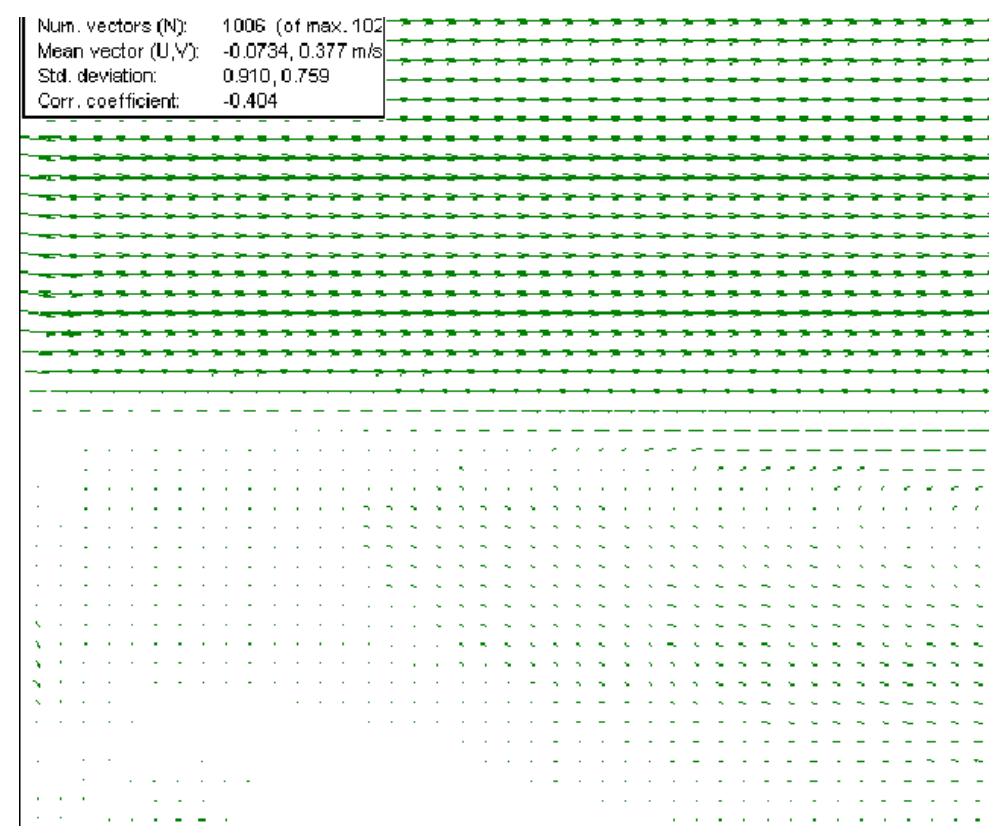

Figure 4. Sample PIV measurement behind a step in the aerosol wind tunnel. 


\section{Module IV, Applications}

The applications module concerns with a number of examples from air pollutions to xerography. Figure 5 shows the photo of the Peace Bridge area in the south west Buffalo, NY. Figure 6 shows a sample computational result for the dispersion of particulate emission form the traffic on the Peace Bridge.

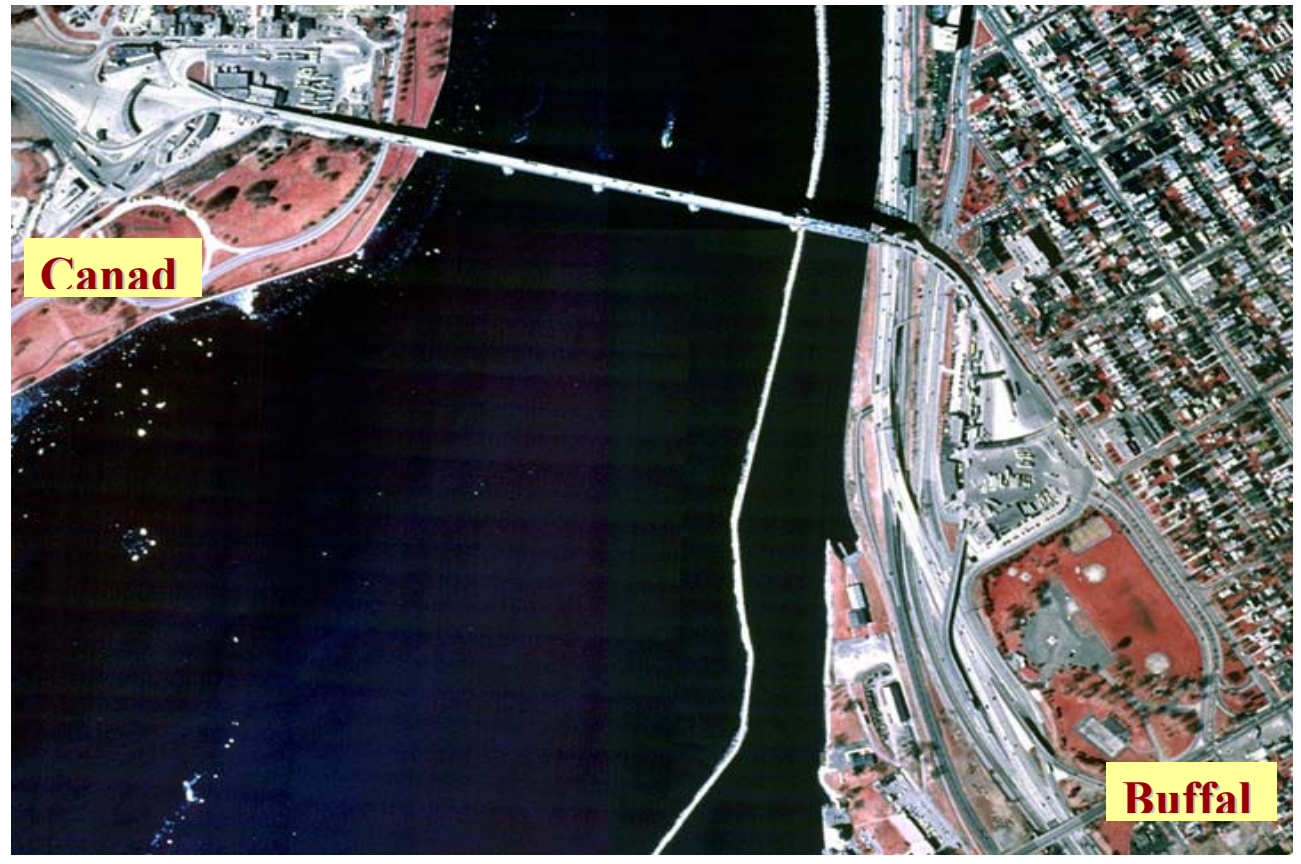

Figure 5. A picture of Peace Bridge area and city of Buffalo.

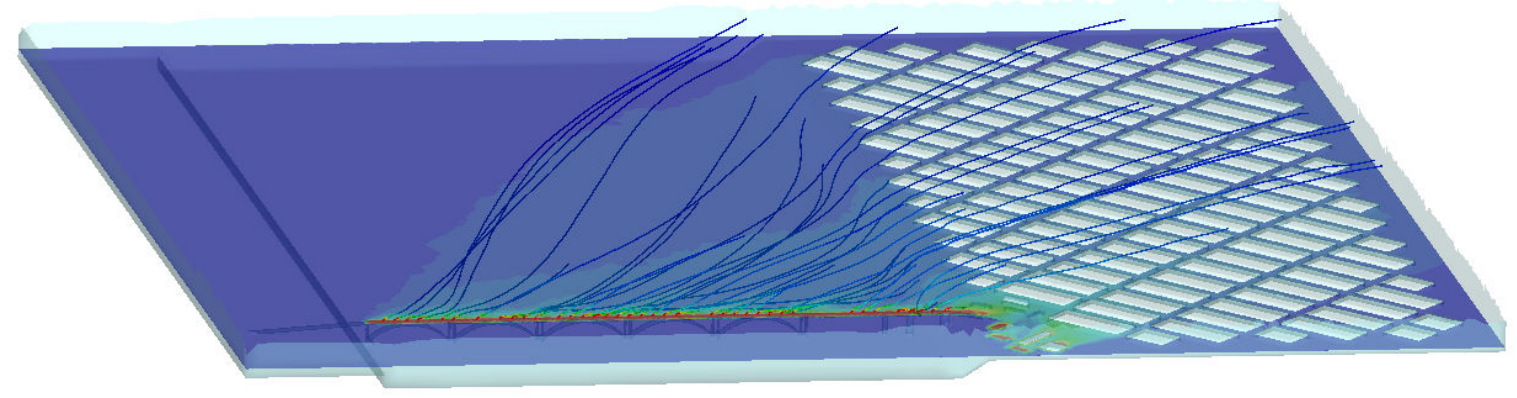

Figure 6. Sample computational result for pollutant dispersion form Peace Bridge traffics.

\section{Conclusions}

The development of a sequence of web-based courses on particle transport, deposition and removal was described. Different modules of the course are outline. Availability of the course material and computational module on the web was very helpful to student learning, and students at multiple campuses could take the course simultaneously.

\section{References}

http://www.clarkson.edu/projects/cred/ 